\title{
INTRODUCTION
}

In a previous paper [1], we proposed an original formulation of the global reverberation in a room, based on the solution of the acoustic radiative transfer equation [2]. The objective was to quantify the influence of the scattering properties of the surfaces of the room on the reverberation time. Indeed, we still miss a clear relation between the scattering coefficients and the reverberation, though some recent works have brought interesting contributions to this problem [3-6].

The results obtained in our previous paper are summarized in the next section. The formulation has been restricted to rooms having specular and diffuse reflections, but with special conditions imposed on the "cloud" of image sources (isotropy and uniformity). The formulation is extended in this paper to rooms having a pair of parallel surfaces, in which a certain degree of anisotropy can be introduced in the cloud of image sources.

\section{SUMMARY OF PREVIOUS RESULTS}

Our formulation is derived from the acoustic radiative transfer equation, and more particularly from its formulation proposed by Navarro et al [2]:

$$
\frac{\partial w(\vec{r}, t)}{\partial t}+\nabla \cdot \vec{J}(\vec{r}, t)+m c w(\vec{r}, t)=q_{0}(\vec{r}, t)
$$

In this equation, $w(\vec{r}, t)$ is the sound energy density $\left(\mathrm{J}_{\mathrm{m}} \mathrm{m}^{-3}\right)$ at position $\vec{r}$ in the room and at time $t . \vec{J}$ is the sound energy flow vector $\left(\mathrm{W} . \mathrm{m}^{-2}\right)$ defined as [1,2] the sum (integral) of all oriented flux of sound particles converging at position $\vec{r}$ in time $t(\vec{J}=0$ for a perfectly diffuse sound field). Finally, $m$ is the attenuation of sound energy in the medium $\left(\mathrm{m}^{-1}\right), c$ is the speed of sound and $q_{0}$ is the sources' power density $\left(\mathrm{W} . \mathrm{m}^{-3}\right)$.

If equation (1) is integrated on the whole volume of the room $V$, then:

$$
V \frac{d \bar{w}(t)}{d t}+\int_{S} \vec{J}\left(\vec{r}_{b}, t\right) \cdot \vec{n}_{x} d S+m c V \bar{w}(t)=W(t)
$$

where $\bar{w}(t)$ is the volume-averaged energy density, $S$ is the surface enclosing the volume $V$ and $\vec{n}_{x}$ is the unit vector normal to this surface at position $\vec{r}_{b} \in d S$ and directed towards the exterior of $V$. Equation (2) simply expresses that the instantaneous variation of the total sound energy in the room is the result of the total power $W(t)$ generated in the room at time $t$ diminished by the power absorbed by the surfaces and the medium. The absorption and scattering properties of the surfaces are included in the boundary conditions [1].

It was shown in [1] that the reverberation decays in rooms with diffusely reflecting boundaries are approximately exponential, after an initial transient period. In a reverberation experiment, if the source is cut off at $t=0$ and $t_{a}$ is the duration of this transient period:

$$
w(\vec{r}, t)=w\left(\vec{r}, t_{a}\right) e^{-\gamma\left(t-t_{a}\right)} \quad \text { and } \quad \bar{w}(t)=\bar{w}\left(t_{a}\right) e^{-\gamma\left(t-t_{a}\right)} \quad t \geq t_{a}>0
$$

The constant $\gamma$ of this exponential decay has been assumed to be the same at all positions in the room. It depends on the absorption coefficients $\alpha\left(\vec{r}_{b}\right)$, on the absorption of the medium and on the relative distribution of the sound energy on the surfaces at time $t_{\mathrm{a}}$ :

$$
\gamma=m c+\frac{c}{4 V} \int_{S} \chi\left(\vec{r}_{b}\right) \frac{w\left(\vec{r}_{b}, t_{a}\right)}{\bar{w}\left(t_{a}\right)} d S \quad \chi\left(\vec{r}_{b}\right)=\frac{\alpha\left(\vec{r}_{b}\right)}{1-\alpha\left(\vec{r}_{b}\right) / 2}
$$


A first improvement of this model has been the introduction of mixed specular and diffuse reflections. In this case, two groups of sound particles exist at each position in the room and at each instant $t$ : those which have already undergone at least one diffuse reflection (the "diffuse" particles $w_{d}$ ) and the others (the "specular" particles $w_{s}$ ):

$$
w(\vec{r}, t)=w_{s}(\vec{r}, t)+w_{d}(\vec{r}, t)
$$

At each reflection of a group of specular particles, their incident flux is partly absorbed $(\alpha)$, partly specularly reflected ((1-s) (1- $\alpha))$ and partly diffusely reflected (s $(1-\alpha))$, where $s$ is the scattering coefficient of the surface at the reflection point. Diffuse particles are never reflected as specular particles in this model.

The contribution $w_{s}(\vec{r}, t)$ in (5) is the sound field generated by the "cloud" of image sources and, therefore, it satisfies equation (1). As the total sound field $w(\vec{r}, t)$ also satisfies (1), so does $w_{d}(\vec{r}, t)$ by (5). Two assumptions were made on the "cloud" of image sources in [1]:

- it must be approximately isotropic,

- $\quad$ it must be the same at all positions $\vec{r}_{b}$ on the room' surface (cloud's uniformity).

Clearly, this brings some restrictions on the kind of rooms that can be represented by the model and this is discussed in the following section.

\section{ISOTROPIC AND UNIFORM CLOUDS OF IMAGE SOURCES}

The assumption of (quasi-) isotropy allows to develop the oriented flux of specular particles $\vec{L}_{s}(\vec{r}, \vec{s}, t)$ (in $\mathrm{W} \cdot \mathrm{m}^{-2} \cdot \mathrm{sr}^{-1}$ ) as a first order spherical harmonics expansion at position $\vec{r}$ and in the direction of the unit vector $\vec{s}$ (see eq. 28 of [2]) :

$$
\vec{L}_{s}(\vec{r}, \vec{s}, t) \cong\left(c w_{s}(\vec{r}, t)+3 \vec{J}_{s}(\vec{r}, t) . \vec{s}\right) \frac{\vec{s}}{4 \pi}
$$

This approximation can be used to derive the specular flux absorbed at any position $\vec{r}_{b}$ on the surface of the room:

$$
\int_{\Omega_{\text {inc }}} \alpha_{S}\left(\vec{r}_{b}\right) \vec{L}_{s}\left(\vec{r}_{b}, \vec{s}, t\right) \cdot \vec{n}_{x} d \Omega=\vec{J}_{s}\left(\vec{r}_{b}, t\right) \cdot \vec{n}_{x} \cong \frac{c}{4} w_{s}\left(\vec{r}_{b}, t\right) \chi_{s}\left(\vec{r}_{b}\right)
$$

In this expression, $\Omega_{\text {inc }}$ is the solid angle containing all incident directions at position $\vec{r}_{b}, \alpha_{s}$ is the specular absorption coefficient $\left(\alpha_{s}=\alpha+s(1-\alpha)\right)$ and $\chi_{s}=\frac{\alpha_{s}}{1-\alpha_{s} / 2}$.

The assumption of (quasi-) isotropy of the cloud of image sources can be considered as a good approximation in rooms where there's no significant anisotropy in the geometry and in the distribution of the acoustical properties on the surfaces. It is also expected that this assumption must be a better approximation at greater distances from the real source, where the number of image sources increases and the local influences of the first-orders image sources are no longer effective.

To verify this, we analyze the sound pressure level distribution (related to $w_{s}$ in (6)) and the amplitude of the sound intensity vector (related to $\left|\vec{J}_{s}\right|$ ) in a cubic room with specularly reflecting surfaces. For example, figure 1 shows the distribution of the ratio $\beta=\frac{c w_{s}(\vec{r}, t)}{\left|\vec{J}_{s}(\vec{r}, t)\right|}$ in $\mathrm{dB}$, computed by ray tracing in a cubic room (length = 10m) equipped with a point source in its center. All (specular) reflection coefficients are identical $\left(\rho_{\mathrm{s}}=0.7\right)$. These ratio $\beta$ 
are computed at mid-height, $50 \mathrm{~ms}$ after the source's cut off time, during a reverberation decay. In this case, the ratio $\beta$ are greater than $9 \mathrm{~dB}$ (a factor 8 ) and the minimum value of $\beta$ is obtained in the vicinity of the room' surfaces.

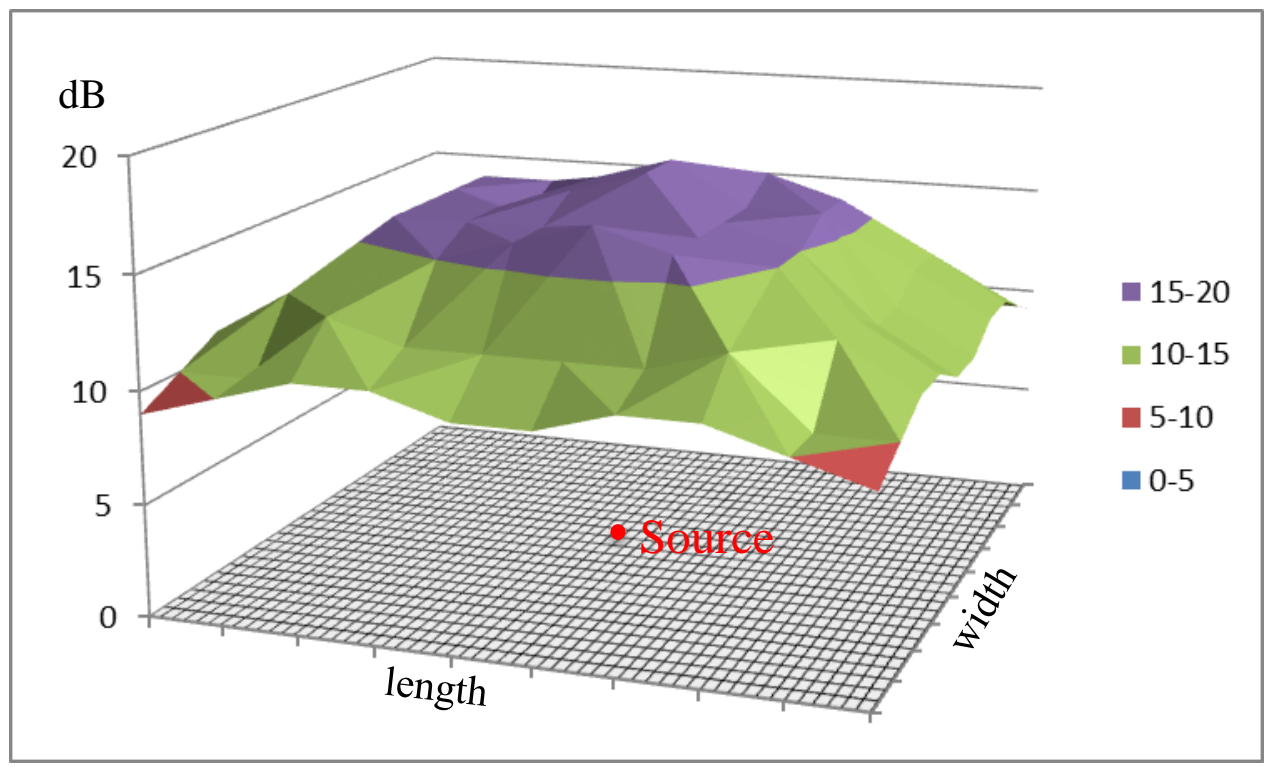

FIGURE 1. Ratio $\beta$ (see definition in the text) between the sound pressure level and the sound intensity vector in $\mathrm{dB}$ computed after 50ms during the reverberation decay, in a cubic room (length $=10 \mathrm{~m}$ ) at 10x10 positions $\vec{r}$ regularly spaced and situated in the mid-height horizontal plane. The sound source occupies a central position in this plane. The reflection coefficient is the same for all surfaces, i.e. $\rho_{\mathrm{s}}=0.7$.

Several combinations of reflection coefficients and receptor's positions have been simulated in this cubic room. The analysis of the computations' results led to the following conclusions:

- if all reflection coefficients are identical, the ratio $\beta(\vec{r}, t)$ is maximum at the room's center and minimum along the surfaces, during a reverberation decay, if $t>50 \mathrm{~ms}$. Table 1 gives the minimum value of this ratio as a function of the reflection coefficient;

- this table suggests that the assumption (6) of the (quasi-) isotropy of the cloud of image sources is reasonable in this cubic room if $\rho_{s} \geq 0.5$ and $t>50 \mathrm{~ms}$. The validity of the assumption degrades as the reflection coefficient decreases;

- if one wall has a different coefficient $\left(\rho_{s w}\right)$ than the other walls $\left(\rho_{s a}\right)$, the validity of the assumption tends to be confirmed if $\rho_{s w} \geq \rho_{s a}$ and it is degraded otherwise. Also, the validity tends to increase with the time $t$ after the source's cut off;

- the sound pressure level is nearly constant (variations less than $1 \mathrm{~dB}$ ) in the whole volume of the room, at all $t>50 m s$ during the reverberation decay.

TABLE 1. Minimum value of the ratio $\beta(\mathrm{dB})$ in the whole volume of the cubic room, for $t>50 \mathrm{~ms}$ after the source's cut off time in a reverberation experiment.

\begin{tabular}{cc}
\hline Reflection coefficient & $\operatorname{Min}_{\vec{r}}\{\beta(\vec{r}, t)\}(\mathrm{dB})$ \\
\hline 0.9 & 14 \\
0.7 & 9 \\
0.5 & 6 \\
0.3 & 4 \\
\hline
\end{tabular}


This last conclusion indicates that it makes sense to assume a "uniform" cloud of image sources in this kind of rooms, meaning that the specular contribution is nearly constant at each position in the room and at each instant, after an "initial" transient time $t_{b}$. We can therefore assume in (7) that $w_{s}\left(\vec{r}_{b}, t\right) \cong \bar{w}_{s}(t)$ if $t>t_{b}$.

With both assumptions (isotropy and uniformity), it was shown in [1] that:

$$
\begin{aligned}
& \bar{w}_{s}(t)=\bar{w}_{s}\left(t_{b}\right) e^{-\gamma_{s}\left(t-t_{b}\right)} \quad t \geq t_{b}>0 \\
& \gamma_{s}=m c+\frac{c}{4 V} \int_{S} \chi_{s}\left(\vec{r}_{b}\right) d S
\end{aligned}
$$

The contributions $\bar{w}_{s}(t)$ and $\bar{w}_{d}(t)$ satisfy the following equations (cfr. [1]):

$$
\begin{aligned}
& \frac{d \bar{w}_{s}(t)}{d t}+\gamma_{s} \bar{w}_{s}(t)=0 \quad t \geq t_{b}>0 \\
& \frac{d \bar{w}_{d}(t)}{d t}+\gamma_{d} \bar{w}_{d}(t)=K_{s} \bar{w}_{s}(t) \quad t \geq t_{b}>0 \\
& K_{s}=\frac{c}{4 V} \int_{S}\left(\chi_{s}-\chi\right)_{\vec{r}_{b}} d S
\end{aligned}
$$

In the second equation, the constant $\gamma_{d}$ has the same definition as in (4), except that $w$ is replaced by $w_{d}$.

\section{ROOMS HAVING A PAIR OF PARALLEL SURFACES}

Let $A_{1}$ and $A_{2}$ represent a pair of parallel surfaces (see figure 2). $A_{1}^{\prime}$ and $A_{2}^{\prime}$ are sections of $A_{1}$ and $A_{2}$ respectively that define the larger prism $\mathscr{P}$ connecting $A_{1}$ to $A_{2}$ and having its lateral faces perpendicular to $A_{1}$ and $A_{2}$.

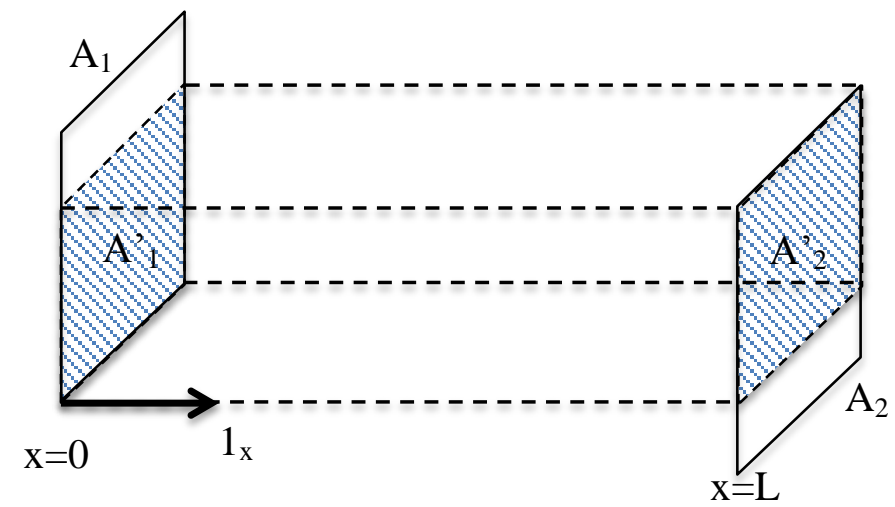

FIGURE 2. The prism $\mathscr{P}$ is the volume contained between surfaces $A_{{ }_{l}}{ }_{l}$ and $A^{\prime}{ }_{2}$, which are sections of the parallel surfaces $A_{l}$ and $A_{2}$ respectively. The axis $\overrightarrow{1}_{x}$ is perpendicular to both surfaces and it is parallel to the faces of the prism. 
We can extract from the cloud of image sources those which are only created by reflections on $A_{1}$ and $A_{2}$. Their contribution is called $w_{p}(\vec{r}, t)$ in the following, whereas $w_{s}(\vec{r}, t)$ is the contribution of the remaining image sources:

$$
\begin{aligned}
& w(\vec{r}, t)=w_{s}(\vec{r}, t)+w_{p}(\vec{r}, t)+w_{d}(\vec{r}, t) \\
& \vec{J}(\vec{r}, t)=\vec{J}_{s}(\vec{r}, t)+\vec{J}_{p}(\vec{r}, t)+\vec{J}_{d}(\vec{r}, t)
\end{aligned}
$$

The larger prism $\mathscr{P}$ is not empty and it is also assumed that the sound source is included in $\mathscr{P}$. We still consider in the following a reverberation experiment, the source being cut off at $t=0$.

The image sources that contribute to $w_{p}$ and $\vec{J}_{p}$ belong to two groups: those which are situated at $x<0$ (see figure 2) and create a flow vector $\vec{J}_{p}^{+}$(quasi-) parallel to $\overrightarrow{1}_{x}$ and those situated at $x>L$ and create a flow vector $\vec{J}_{p}^{-}$(quasi-) parallel to $-\overrightarrow{1}_{x}$. Similarly, we have:

$$
w_{p}(\vec{r}, t)=w_{p}^{+}(\vec{r}, t)+w_{p}^{-}(\vec{r}, t) \quad \vec{r} \in \mathscr{P}
$$

If the geometrical divergence between $(c t)$ and $(c t+2 L)$ is neglected (this can be done in a reverberation experiment if $t$ is "great enough"), then we have:

$$
w_{p}^{ \pm}\left(x, t+\frac{2 L}{c}\right) \cong w_{p}^{ \pm}(x, t) \rho_{s 1} \rho_{s 2} e^{-2 m L} \quad t>>0
$$

where $\rho_{s 1}$ and $\rho_{s 2}$ are the specular reflection coefficients of $A_{1}$ and $A_{2}$ respectively. Note that (12) is an asymptotic expression, valid if $t>>0$. This approximation is also equivalent to the assumption of plane waves travelling in the $\pm \overrightarrow{1}_{x}$ direction between the surfaces $A_{l}$ and $A_{2}$. From (12), we have:

$$
\begin{aligned}
& w_{p}^{ \pm}(x, t) \cong W_{p}^{ \pm}(x) e^{-\gamma_{12} t} \quad t>0 \quad \text { and } \quad 0 \leq x \leq L \\
& \gamma_{12}=-\frac{c}{2 L} \ln \left(\rho_{s 1} \rho_{s 2} e^{-2 m L}\right)
\end{aligned}
$$

Similar approximations are obtained for the sound energy flow vectors, since :

$$
\vec{J}_{p}^{ \pm}(x, t)= \pm c w_{p}^{ \pm}(x, t) \overrightarrow{1}_{x}
$$

Now, the radiative transfer equation (2) applies for the three contributions $\left(\bar{w}_{p}, \bar{w}_{s}, \bar{w}_{d}\right)$ individually, with $W(t)=0$ for $t>0$ in a reverberation experiment. We still consider that the flux of specular particles reflected by any $d S$ is only created by the incident flux of specular particles. Moreover, we introduce a further approximation, i.e. that the transfer of energy during a reflection from $\vec{J}_{p}$ to $\vec{J}_{s}$ can be neglected. This is again equivalent to neglect the geometrical divergence of the flux $\vec{J}_{p}$ and assume that it is only incident on $A^{\prime}{ }_{1}$ and $A^{\prime}{ }_{2}$. Of course, there exists no transfer of energy from $\vec{J}_{s}$ to $\vec{J}_{p}$ and the scattering coefficient of $d S$ still allows transfers from $\vec{J}_{s}$ and $\vec{J}_{p}$ to $\vec{J}_{d}$. 
If the cloud of image sources giving the contribution $w_{s}$ is still (quasi-) isotropic and uniform, then $\bar{w}_{s}(t)$ still satisfies (8). Furthermore, $\bar{w}_{p}(t)$ is obtained by averaging (13) on the whole volume of the room (not only the prism $\mathscr{P})$, which gives:

$$
\bar{w}_{p}(t) \cong \frac{A_{1}^{\prime}}{V} e^{-\gamma_{12} t} \int_{0}^{L}\left(W_{p}^{+}(x)+W_{p}^{-}(x)\right) d x \quad t>>0
$$

Solving equation (2) for $\bar{w}_{d}(t)$ is somewhat more complex, since we must take into account the diffuse energy created by the scattering of both specular fluxes on the surfaces of the room. The flux $\left(\vec{J}_{d} \cdot \vec{n}_{x}\right)$ in (2) is given by (see eq. (7)):

$$
\vec{J}_{d}\left(\vec{r}_{b}, t\right) \cdot \vec{n}_{x}=\int_{\Omega_{\text {irc }}} \alpha\left(\vec{r}_{b}\right) \vec{L}_{d}\left(\vec{r}_{b}, \vec{s}, t\right) \cdot \vec{n}_{x} d \Omega-\phi_{\text {scat }}\left(\vec{r}_{b}\right)
$$

The oriented flux of diffuse sound particles $\vec{L}_{d}$ is assumed to be (quasi-) isotropic as in [1]. Therefore, it can be developed as a first order spherical harmonics expansion at position $\vec{r}_{b}$, which gives (see eq. (6)):

$$
\left(1-\frac{\alpha\left(\vec{r}_{b}\right)}{2}\right) \vec{J}_{d}\left(\vec{r}_{b}, t\right) \cdot \vec{n}_{x}=\frac{c \alpha\left(\vec{r}_{b}\right)}{4} w_{d}\left(\vec{r}_{b}, t\right)-\phi_{s c a t}\left(\vec{r}_{b}, t\right)
$$

The flux $\phi_{\text {scat }}\left(\vec{r}_{b}, t\right)$ is the part of the incident specular flux that is scattered at position $\vec{r}_{b}$ at time $t$. The contribution of $\vec{J}_{p}$ to this flux is:

$$
\begin{array}{ll}
s_{1}\left(\vec{r}_{b}\right)\left(1-\alpha_{1}\left(\vec{r}_{b}\right)\right) c W_{p}^{-}(x=0) e^{-\gamma_{12} t} & \text { if } \vec{r}_{b} \in A_{1}^{\prime} \\
s_{2}\left(\vec{r}_{b}\right)\left(1-\alpha_{2}\left(\vec{r}_{b}\right)\right) c W_{p}^{+}(x=L) e^{-\gamma_{12} t} & \text { if } \vec{r}_{b} \in A^{\prime}{ }_{2} \\
\cong 0 \quad \text { if } \vec{r}_{b} \notin\left\{A_{1}^{\prime}, A_{2}^{\prime}\right\} &
\end{array}
$$

The contribution of $\vec{J}_{s}$ to this flux can be derived from (7):

$$
s\left(\vec{r}_{b}\right)\left(1-\alpha\left(\vec{r}_{b}\right)\right) \frac{1}{\alpha_{s}\left(\vec{r}_{b}\right)} \vec{J}_{s}\left(\vec{r}_{b}, t\right) \cdot \vec{n}_{x} \cong \frac{c}{4} \frac{s\left(\vec{r}_{b}\right)\left(1-\alpha\left(\vec{r}_{b}\right)\right)}{1-\alpha_{s}\left(\vec{r}_{b}\right) / 2} w_{s}\left(\vec{r}_{b}, t\right)
$$

Finally, eq. (2) for $\bar{w}_{d}(t)$ is derived from (4), (8) and (17-19):

$$
\frac{d \bar{w}_{d}(t)}{d t}+\gamma_{d} \bar{w}_{d}(t)=K_{s d} \bar{w}_{s}(t)+K_{12} \bar{w}_{p}(t) \quad t>>0
$$

The constant $\gamma_{d}$ is the same as in (9), while $K_{s d}$ and $K_{12}$ depend on the scattering coefficients and they express the transfer of sound energy from the specular to the diffuse contribution. If we introduce the vector of sound energy densities $\vec{w}(t)=\left[\bar{w}_{s}(t), \bar{w}_{p}(t), \bar{w}_{d}(t)\right]$, the transfer equation results in a matrix equation, similar to the SEA formulation that was suggested by [7]: 


$$
\frac{d}{d t} \vec{w}(t)+L \vec{w}(t)=0 \quad t>t_{i n} \quad L=\left(\begin{array}{ccc}
\gamma_{s} & 0 & 0 \\
0 & \gamma_{12} & 0 \\
-K_{s d} & -K_{12} & \gamma_{d}
\end{array}\right)
$$

This equation is valid for $t>t_{i n}$, the "initial" transient time necessary for all assumptions that have been formulated for all components of the vector $\vec{w}(t)$ to be fulfilled. The solution of (21) is :

$$
\vec{w}(t)=e^{-L\left(t-t_{i n}\right)} \vec{w}\left(t_{i n}\right) \quad t>t_{i n}
$$

\section{DISCUSSION ON POSSIBLE APPLICATIONS}

As such, the model (22) is not particularly suited for a simple formulation of a relation between the reverberation time in a room and the scattering coefficients of its surfaces. First of all, the constants $K_{s d}$ and $K_{12}$ are complex expressions of the scattering coefficients: some simplifications could be introduced (for example in rooms where the absorption is low), but this would introduce new assumptions and reduce the scope of the model. Second, we need to know the distribution of the sound energy densities $\left\lfloor\bar{w}_{s}, \bar{w}_{p}, \bar{w}_{d}\right\rfloor$ at $t_{i n}>0$, and this is far from being obvious.

Third, the solution of (22) is a sum of three exponential decays and the corresponding reverberation time of such a decay has no simple expression, except if the three slopes are approximately identical.

However, the model (22) of the sound field can be used in combination with a sound particles or ray tracing algorithm to speed up the computation of the reverberation decay. These algorithms can indeed be applied to:

- compute the echograms between $t=0$ and $t=t_{i n}$, taking into account the exact geometrical description of the room and the real distribution of the absorption and scattering coefficients on the surfaces,

- compute the remaining sound energy densities $\left[\bar{w}_{s}, \bar{w}_{p}, \bar{w}_{d}\right\rfloor$ at $t_{i n}$, the initial conditions of the solution (22).

Then, the matrix $L$ can be evaluated and the reverberation decays are completed for $t>t_{\text {in }}$ through (22).

\section{REFERENCES}

1. J.J. Embrechts, "Searching for a theoretical relation between reverberation and the scattering coefficients of surfaces in a room," on the CD-ROM: Proceedings of the Acoustics 2012 Nantes Conference, $11^{\text {th }}$ French CFA congress and 2012 Annual IOA Meeting, 2397-2402 (2012).

2. J.M. Navarro, F. Jacobsen, J. Escolano, and J.J. Lopez, "A theoretical approach to room acoustic simulations based on a radiative transfer model," Acta Acustica united with Acustica 96, 1078-1089 (2010).

3. T. Hanyu, "A theoretical framework for quantitatively characterizing sound field diffusion based on scattering coefficient and absorption coefficient of walls," J. Acoust. Soc. Am. 128, 1140-1148 (2010).

4. H. Onaga, and J.H. Rindel, "Acoustic characteristics of urban streets in relation to scattering caused by building facades," Applied Acoustics 68, 310-325 (2007).

5. D. Sumarac-Pavlovic, and M. Mijic, "An insight into the influence of geometrical features of rooms on their acoustic response based on free path length distribution," Acta Acustica united with Acustica 93, 1012-1026 (2007).

6. T. Sakuma, "Approximate theory of reverberation in rectangular rooms with specular and diffuse reflections," J. Acoust. Soc. Am. 132, 23252336 (2010).

7. L.I. Wilmhurst, and D.J. Thompson, "A method for predicting the reverberation time and decay curves of rectangular rooms with non-uniform absorption distribution using Statistical Energy Analysis," on the CD-ROM: Proceedings of the Acoustics 2012 Nantes Conference, $11^{\text {th }}$ French CFA congress and 2012 Annual IOA Meeting, 1429-1434 (2012). 